\title{
Studying the jovian Trojan dust
}

\author{
G. C. de Elía and A. Brunini \\ Facultad de Ciencias Astronómicas y Geofísicas, Universidad Nacional de La Plata, Paseo del Bosque S/N (1900), La Plata, Argentina \\ IALP-CCT La Plata-CONICET \\ e-mail: [gdeelia; abrunini] @fcaglp.unlp.edu.ar
}

Received 6 March 2009 / Accepted 8 December 2009

ABSTRACT

\begin{abstract}
Aims. We analyze the jovian Trojan dust in the $L_{4}$ swarm.
Methods. To do this, we use a modification of the numerical code developed by us and previously applied to the collisional and dynamical evolution of the $L_{4}$ jovian Trojans. This algorithm considers catastrophic collisions and cratering events, includes a dynamical treatment that takes the stability and instability zones of the $L_{4}$ jovian swarm into account, and incorporates the effects of the Poynting-Robertson radiation.

Results. From this analysis, we infer that the time evolution of the $L_{4}$ jovian Trojan dust luminosity has the characteristics of a diffusion process, since its current value is fairly insensitive to variations in the initial size distribution. Moreover, our results indicate that the current luminosity of the dust in the $L_{4}$ jovian swarm ranges from $\sim 3.2 \times 10^{-8}$ to $3.4 \times 10^{-8} L_{\odot}$.

Conclusions. From these estimates, we conclude that the current luminosity of the dust in the $L_{4}$ jovian swarm is comparable to the luminosity of the inner Solar System dust, and, at least, one order of magnitude lower than the luminosity of the outer Solar System dust.
\end{abstract}

Key words. minor planets, asteroids: general - methods: numerical - planets and satellites: formation

\section{Introduction}

The jovian Trojan asteroids are objects locked in a 1:1 mean motion resonance with Jupiter, which lead and trail the planet by $60^{\circ}$ of longitude, librating around the Lagrangian equilibrium points $L_{4}$ and $L_{5}$. The collisional processes have played a significative role in the evolution of the jovian Trojans (Davis \& Weidenschilling 1981; Milani 1993, 1994; Marzari et al. 1997; de Elía \& Brunini 2007). The collisions among these objects produce several dust particles whose lifetimes are affected primarily by radiation mechanisms (Burns et al. 1979).

The existence of dust in the inner Solar System has long been known from observations of the zodiacal light, which was first investigated by the astronomer Giovanni Domenico Cassini in 1683. In the second half of the twenty century, the existence of dust particles was confirmed in situ by detectors on the Pioneer 10 and 11, Voyager, Galileo, and Ulyses spacecrafts, while the IRAS and COBE space telescopes observed the thermal emission of the zodiacal cloud. Direct measurements of the terrestrial mass accretion rate of cosmic dust indicate that the dust particle diameter ranges from about 5 to $500 \mu \mathrm{m}$, with a strong peak near $220 \mu \mathrm{m}$ (Love \& Brownlee 1993). In the outer Solar System, the dust particles were detected in situ by the Pioneer 10 and 11 spacecrafts beyond the orbit of Saturn (Landgraf et al. 2002), but the IRAS and COBE space telescopes were unable to detect this thermal emission because of the zodiacal light.

In this work, we analyze the jovian Trojan dust in the $L_{4}$ swarm, produced by particles with diameters $D \sim 5-500 \mu \mathrm{m}$. We decided to focus our discussion on the $L_{4}$ swarm since the present work represents a continuation of a previous paper (de Elía \& Brunini 2007) concerning the collisional and dynamical evolution of the $L_{4}$ jovian Trojans. Here, we determine the area of the dust cloud in the $L_{4}$ jovian swarm over
4.5 Gyr of evolution. Then, we calculate its fractional luminosity $L_{\text {Dust }} / L_{\odot}$ and compare it with the estimates of the dust thermal emission in the inner and outer parts of the Solar System.

\section{The full model}

To simulate the collisional and dynamical evolution of the jovian Trojans of the $L_{4}$ swarm, we make our numerical code evolve the number of bodies residing in a set of 130 discrete logarithmic size bins with time. The central values of these size bins range from $D_{1}=10^{-10} \mathrm{~km}$ to $D_{130}=886.7 \mathrm{~km}$ in diameter, in such a way that there are 10 size bins for each factor of 10 in diameter. Thus, from one bin to the next, the diameter of the bodies changes by a factor of $2^{1 / 3}$ and the mass changes by a factor of 2 . Moreover, our model adopts a density of $1.5 \mathrm{~g} \mathrm{~cm}^{-3}$, suggesting that the jovian Trojans are more porous and have a lower density than S-type asteroids.

Following Campo Bagatin et al. (1994) and Campo Bagatin (1998), a collisional system with a low-mass cutoff leads to waves in the size distribution of the bodies. To avoid this effect and to analyze the behavior of $D \sim 5-500 \mu \mathrm{m}$ particles, the current version of the code does not evolve the 17 first size bins with time, whose central values range from 0.1 to $4 \mu \mathrm{m}$. In fact, this part of the population is only used as a tail of projectiles for calculating impact rates with larger bodies, and its size distribution is determined each timestep by extrapolating the slope of the distribution of the 10 next size bins.

In the following, we present the initial populations, the collisional parameters, and the main dynamical considerations of our model. Then, we give a brief description of the collisional and dynamical numerical algorithm, discussing how the radiation forces affect the small particles. 


\subsection{Initial populations}

The size distribution of the $L_{4}$ jovian Trojans has been studied by Jewitt et al. (2000). According to this work, the cumulative size distribution of this population is adequately fitted by

$N\left(>D_{0.04}\right)=6.4 \times 10^{5}\left(\frac{1 \mathrm{~km}}{D_{0.04}}\right)^{2.0 \pm 0.3}$

for $4.4 \leq D_{0.04} \leq 40 \mathrm{~km}$, and

$N\left(>D_{0.04}\right)=1.8 \times 10^{10}\left(\frac{1 \mathrm{~km}}{D_{0.04}}\right)^{4.5 \pm 0.9}$

for $D_{0.04} \geq 84 \mathrm{~km}$, where $D_{0.04}$ is the diameter derived by assuming a geometric albedo of 0.04 , which is the mean value of known Jupiter Trojans (Tedesco 1989; Fernández et al. 2003). From this, Jewitt et al. (2000) estimate that the total mass of $L_{4}$ jovian Trojans is about $5 \times 10^{23} \mathrm{~g}\left(\sim 9 \times 10^{-5} M_{\oplus}\right)^{1}$. On the other hand, taking the analysis of Binzel \& Sauter (1992) concerning the lightcurve amplitude distribution of jovian Trojan asteroids into account, Jewitt et al. (2000) suggest that a primordial/fragment transition occurs at a diameter near $60-80 \mathrm{~km}$. From this argument, we adopt the following cumulative starting population

$N(>D)=C\left(\frac{1 \mathrm{~km}}{D}\right)^{p}$ for $D \leq 60 \mathrm{~km}$,

$N(>D)=6 \times 10^{10}\left(\frac{1 \mathrm{~km}}{D}\right)^{4.7}$ for $D>60 \mathrm{~km}$,

(de Elía \& Brunini 2007) where $C=6 \times 10^{10}(60)^{-4.7}(60)^{p}$ by continuity for $D=60 \mathrm{~km}$, and $p$ is a given cumulative power-law index. To discuss the dependence of our simulations on the initial mass of the population, here, we decided to use two different initial populations, which are defined as

- initial Population 1, which assumes $p=2.1$, leading an initial mass of $\sim 1.2$ times the current $L_{4}$ jovian swarm mass;

- initial Population 2, which assumes $p=3.1$, leading an initial mass of $\sim 108$ times the current $L_{4}$ jovian swarm mass.

These initial cumulative size distributions are shown in Fig. 1, along with the observational data derived by Jewitt et al. (2000) and the fit proposed by these authors. Yoshida \& Nakamura (2005), Szabó et al. (2007), and Yoshida \& Nakamura (2008) developed studies concerning the size distribution for small jovian Trojans, which are consistent with Jewitt et al. (2000).

\subsection{Collisional parameters}

There are three fundamental quantities for any collisional evolution study:

- the mean impact velocity $\langle V\rangle$;

- the intrinsic collision probability $\left\langle P_{i}\right\rangle$; and

- the impact strength law of the colliding bodies.

In this work, we adopt constant values of the mean impact velocity $\langle V\rangle$ and the intrinsic collision probability $\left\langle P_{i}\right\rangle$ for jovian Trojans derived by the Dell' Oro et al. (1998) algorithm, which takes the resonant behavior of bodies into account. Based on a sample of 223 Jupiter Trojans, these authors computed values of

\footnotetext{
1 Jewitt et al. (2000) assumed a density of $2 \mathrm{~g} \mathrm{~cm}^{-3}$ for the $L_{4}$ jovian Trojans.
}

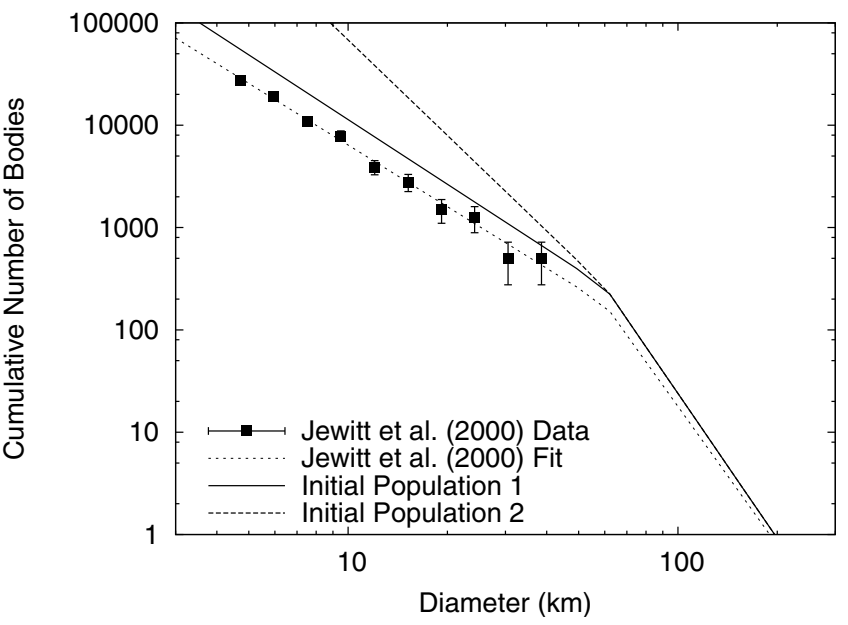

Fig. 1. Initial populations of the model, with observational data derived by Jewitt et al. (2000) and the fit proposed by these authors.

$\langle V\rangle$ and $\left\langle P_{i}\right\rangle$ of $4.66 \mathrm{~km} \mathrm{~s}^{-1}$ and $7.79 \pm 0.67 \times 10^{-18} \mathrm{~km}^{-2} \mathrm{yr}^{-1}$, respectively, for the $L_{4}$ jovian swarm.

Concerning the impact strength, O'Brien \& Greenberg (2005) show that the general shape of the final evolved asteroid population is determined primarily by the impact energy required for dispersal $Q_{\mathrm{D}}$, but variations in the shattering impact specific energy $Q_{\mathrm{S}}$ and the inelasticity parameter $f_{k e}$ can affect such a final population even if $Q_{\mathrm{D}}$ is held the same. According to these arguments, we decided to choose a combination of the parameters $Q_{\mathrm{S}}$ and $f_{k e}$ that yield the $Q_{\mathrm{D}}$ law from Benz \& Asphaug (1999) for icy bodies at $3 \mathrm{~km} \mathrm{~s}^{-1}$. We use a $Q_{\mathrm{D}}$ law for ice rather than for rock since, according to Fornasier et al. (2007), both $L_{4}$ and $L_{5}$ jovian clouds are dominated by D-type asteroids, which are possibly composed of anhydrous silicates, organic compounds, and ice.

In a recent paper concerning the $L_{4}$ jovian Trojans, de Elía \& Brunini (2007) analyze the dependence of their numerical simulations on the shattering impact specific energy $Q_{\mathrm{S}}$. This work indicates that the smallest gaps between $Q_{\mathrm{S}}$ and $Q_{\mathrm{D}}$ curves lead to the smallest wave amplitudes in the size distribution of the final evolved population, as well as to the highest ejection rates of fragments. In fact, de Elía \& Brunini (2007) show that, if the $Q_{\mathrm{S}}$ law has a large gap with respect to the $Q_{\mathrm{D}}$ law, the final size distribution of the $L_{4}$ jovian Trojans is too wavy compared to the observational data derived by Jewitt et al. (2000). Following these arguments, in this work we decided to use only a $Q_{\mathrm{S}}$ law with a small gap with respect to the $Q_{\mathrm{D}}$ law from Benz \& Asphaug (1999) for icy bodies at $3 \mathrm{~km} \mathrm{~s}^{-1}$. This $Q_{\mathrm{S}}$ law agrees closely with the estimates of the impact strength of asteroids proposed by different authors (Farinella et al. 1982; Davis et al. 1985; Housen \& Holsapple 1990; Housen 1991; Holsapple 1994; Ryan \& Melosh 1998; Benz \& Asphaug 1999). Figure 2 shows the $Q_{\mathrm{S}}$ law used in our simulations, as well as the $Q_{\text {D }}$ law from Benz \& Asphaug (1999) for icy bodies at $3 \mathrm{~km} \mathrm{~s}^{-1}$.

Once the $Q_{\mathrm{S}}$ law is specified, we adjust the ineslaticity parameter $f_{k e}$ to get the Benz \& Asphaug (1999) $Q_{\mathrm{D}}$ law. According to O'Brien \& Greenberg (2005), we express the parameter $f_{k e}$ as

$f_{k e}=f_{k e_{0}}\left(\frac{D}{1000 \mathrm{~km}}\right)^{\gamma}$

where $f_{k e_{0}}$ is the value of $f_{k e}$ at $1000 \mathrm{~km}$ and $\gamma$ is a given exponent. Our simulations indicate that the $Q_{\mathrm{D}}$ law from Benz \& Asphaug (1999) for icy bodies at $3 \mathrm{~km} \mathrm{~s}^{-1}$ is obtained with good 


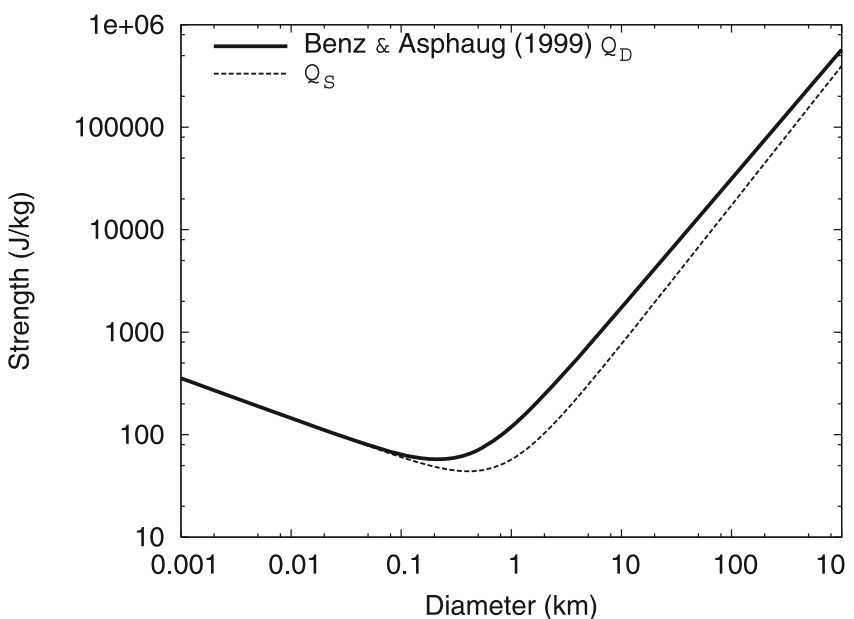

Fig. 2. Impact strength. The dashed line represents the $Q_{\mathrm{S}}$ law used in our simulations. The $Q_{\mathrm{D}}$ law from Benz \& Asphaug (1999) for icy bodies at $3 \mathrm{~km} \mathrm{~s}^{-1}$ is plotted as a solid line.

accuracy from the combination of the selected $Q_{\mathrm{S}}$ law and $f_{k e}$, with $f_{k e_{0}}=0.35$ and $\gamma=0.7$. Such values are consistent with those discussed by Davis et al. (1989).

\subsection{Dynamical considerations}

Figure 3 presents the distribution of 1155 jovian Trojans associated to the $L_{4}$ swarm, with respect to semimajor axis $a$, eccentricity $e$, and inclination $i$. Such plots indicate that all the Jupiter Trojans observed in $L_{4}$ present $a, e$, and $i$ values ranging between 4.7 and $5.7 \mathrm{AU}, 0$ and 0.3 , and 0 and $60^{\circ}$, respectively. In the following, these will be the boundaries of $L_{4}$ in semimajor axis, eccentricity, and inclination with which we perform our work. Assuming that the positions occupied by the asteroids in the planes $(a, e)$ and $(a, i)$ represent stable zones of the swarm, it is possible to define a set of stability and instability niches within the boundaries of the cloud. In fact, we construct such regions assuming widths of $0.02 \mathrm{AU}, 0.0125$, and $2.25^{\circ}$ in semimajor axis, eccentricity, and inclination, respectively. It is worth noting that we select small size niches in order to minimize the influence of the isolated Trojans on the distribution of the population. The stability and instability niches generated from this procedure are indicated in Fig. 3.

\subsection{Numerical algorithm}

Consider a collision between two $L_{4}$ jovian Trojans $T_{1}$ and $T_{2}$ with diameters $D_{T_{1}}$ and $D_{T_{2}}$, respectively. Using the numerical code of de Elía \& Brunini (2007), it is possible to determine

- the size distributions of the fragments from both bodies, which are binned into arrays $F_{T_{1}}$ and $F_{T_{2}}$;

- the velocity distributions of the fragments from both bodies, which are binned into arrays $V_{T_{1}}$ and $V_{T_{2}}$;

- the escape velocity $V_{\text {esc }}$ of the system of two colliding bodies.

Once this information has been computed, the escape and reaccumulation processes of the fragments can be treated. If the fragments in any size bin of the arrays $F_{T_{1}}$ and $F_{T_{2}}$ have a velocity lower than $V_{\text {esc }}$, so they do not escape from the gravitational field of the system and are removed from their respective arrays. Particularly, this occurs with the largest fragment in the case of a
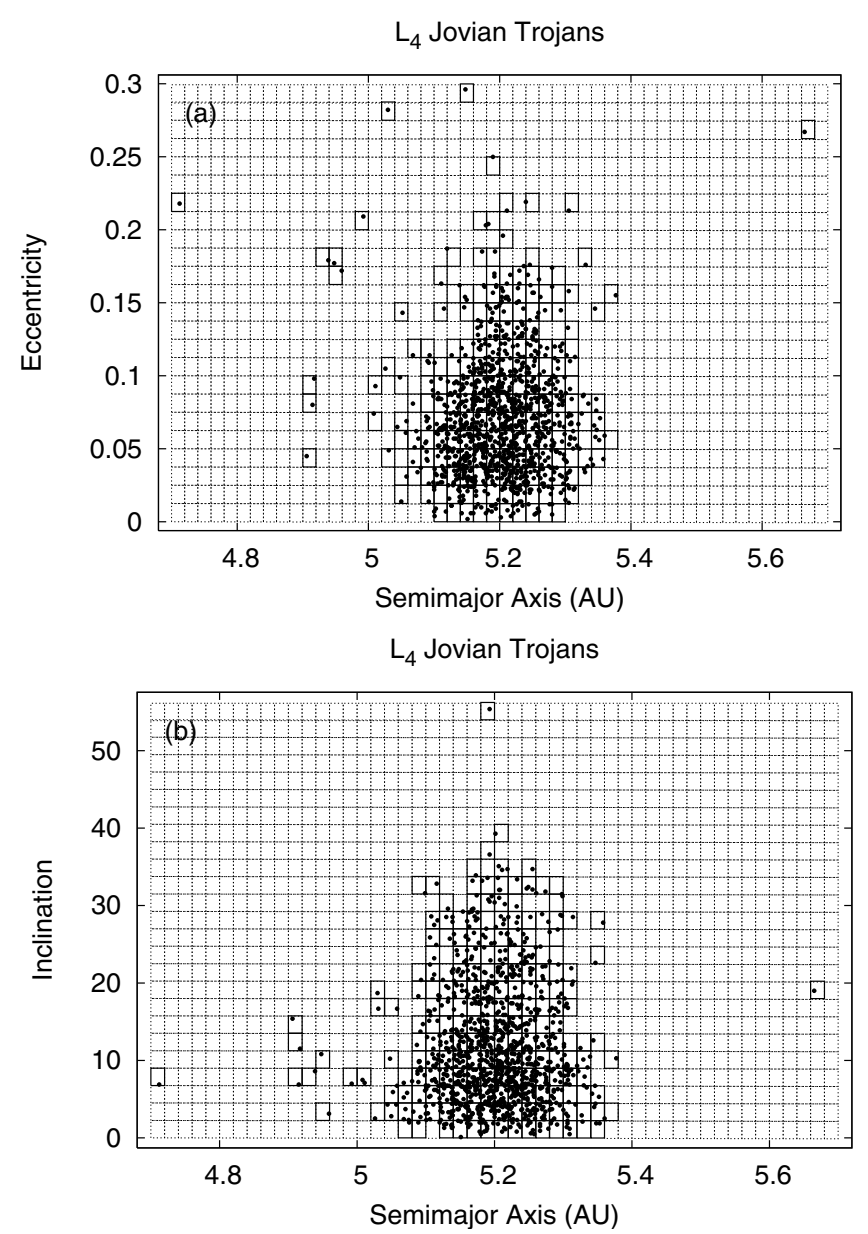

Fig. 3. The distribution of the population of $L_{4}$ jovian Trojans with respect to semimajor axis, eccentricity, and inclination. The solid and dashed squares represent the stability and instability niches used in our simulations, respectively. (Data obtained from http://wWw. cfa. harvard.edu/iau/lists/JupiterTrojans.html)

catastrophic collision and the cratered body in the case of a cratering event, since they are assumed to have a negligible kinetic energy in the center of mass of the system. Then, such fragments are added to the reaccumulated mass below the smallest size bin, producing a single reaccumulated body of diameter $D_{\mathrm{r}}$, belonging to the bin $k_{\mathrm{r}}$ of the $L_{4}$ jovian Trojan size distribution.

In contrast, if the fragments in any size bin of the arrays $F_{T_{1}}$ and $F_{T_{2}}$ have a velocity higher than $V_{\text {esc }}$, so they escape from the gravitational field of the system and remain in their corresponding array. To determine their final fate, we propose a simple method that considers the stability and instability regions of the $L_{4}$ jovian swarm. In each timestep, a characteristic orbit is generated at random for each collision between Trojans $T_{1}$ and $T_{2}$ with diameters $D_{T_{1}}$ and $D_{T_{2}}$, respectively. To do this, we construct 3-D niches within the boundaries of the $L_{4}$ jovian swarm with widths of $0.02 \mathrm{AU}, 0.0125$, and $2.25^{\circ}$ in semimajor axis $a$, eccentricity $e$, and inclination $i$, respectively. From Figs. 3a and b, we computed the normalized distribution of Trojans in each of those niches, which is equivalent to a probability distribution function $f(a, e, i)$. Then, we assigned to our fictitious Trojans orbital elements $a, e, i$, following the probability distribution function $f(a, e, i)$ by J. von Neumann's acceptance-rejection method (Knuth 1981). In mean motion resonances, the evolution of $a, e$ and $i$ is coupled. However, here, they are treated as uncorrelated variables. Nevertheless, a more rigorous treatment would be very 
difficult, and we believe that the results would be not very different from the ones found here. Thus, given uniformly at random, the longitude of ascending node $\Omega$, the argument of pericentre $\omega$, and the mean anomaly $M$ between 0 and $360^{\circ}$, an orbit can be assigned and from this, a position-velocity pair derived for each of the colliding Trojans.

Once has been done, it is necessary to calculate the orbital elements of the fragments once they are ejected from their parent body. Immediately before the collision, the barycentric position and velocity of the fragments are assumed to be those associated with their parent body. After the collision, we consider that the barycentric position of the fragments does not change, while the relative velocities with respect to their parent body are assumed to be equally partitioned between the three components. Once the barycentric position and velocity of the fragments after the collision have been obtained, it is possible to calculate their orbital elements and their final fates. For this, we use the following criteria:

1. The fragments remain in the $L_{4}$ jovian swarm and stay in their respective array $F_{T_{j}}$, if the combinations of $(a, e)$ and $(a, i)$ values are associated with some of the stability niches shown in Figs. $3 a$ and b, respectively, and the absolute value of the difference between their final and initial mean longitudes is smaller than the mean libration amplitude for the $L_{4}$ jovian Trojans, which is assumed to be $\sim 30^{\circ}$ (Marzari et al. 2002; Marzari et al. 2003).

2. On the other hand, the fragments are ejected from the $L_{4}$ jovian swarm and removed from their respective array $F_{T_{i}}$, no longer participating in the collisional evolution, if any of the following conditions is fulfilled:

- eccentricity $e \geq 1$;

- eccentricity $e<1$, but $(a, e, i)$ values exceed the boundaries of the $L_{4}$ swarm;

- eccentricity $e<1$, but $(a, e)$ and $(a, i)$ values are associated with some of the instability niches shown in Figs. 3a and $b$, respectively;

- eccentricity $e<1$, but $(a, e)$ and $(a, i)$ values are associated with some of the stability niches shown in Figs. 3a and $b$, respectively, and the absolute value of the difference between the initial and final mean longitudes is greater than the mean libration amplitude of $\sim 30^{\circ}$.

The frequency of collision between two Trojans $T_{1}$ and $T_{2}$ with diameters $D_{T_{1}}$ and $D_{T_{2}}$, is given by

$f_{T_{1}, T_{2}}=\frac{\left\langle P_{i}\right\rangle}{4}\left(D_{T_{1}}+D_{T_{2}}\right)^{2}$,

where $\left\langle P_{i}\right\rangle$ is the intrinsic collision probability (Sect. 2.2). If $\Delta t$ represents a given time interval and $N_{T_{1}, T_{2}}$ is the number of pairs of Trojans $T_{1}$ y $T_{2}$, the number of collisions during $\Delta t$ can be calculated by

$N_{\mathrm{col}}=f_{T_{1}, T_{2}} N_{T_{1}, T_{2}} \Delta t$.

If both colliding Trojans belong to the same size bin so their numbers $N_{T_{1}}$ and $N_{T_{2}}$ are equal, then $N_{T_{1}, T_{2}}$ adopts an expression of the form

$N_{T_{1}, T_{2}}=\frac{N_{T_{1}}\left(N_{T_{2}}-1\right)}{2}$.

For all other cases, $N_{T_{1}, T_{2}}$ is given by

$N_{T_{1}, T_{2}}=N_{T_{1}} N_{T_{2}}$.
In a given time interval $\Delta t$, the colliding Trojans $T_{1}$ and $T_{2}$ are removed from the size distribution, while the resulting fragments that remain in the $L_{4}$ jovian swarm and the reaccumulated bodies are distributed in the different size bins of the population. Thus, the number of Trojans $T_{1}$ and $T_{2}$ removed from the size distribution during $\Delta t$ is equal to the number of collisions between them, which is given by Eq. (6). This quantity also represents the number of reaccumulated bodies produced from collisions between Trojans $T_{1}$ and $T_{2}$ during $\Delta t$, which are added to the size distribution in their corresponding bin $k_{r}$. On the other hand, the total number of fragments resulting from collisions between Trojans $T_{1}$ and $T_{2}$ during $\Delta t$, which are added to the size distribution in the bin $k$, is given by $\left(F_{T_{1}}^{k}+F_{T_{2}}^{k}\right) f_{T_{1}, T_{2}} N_{T_{1}, T_{2}} \Delta t$. If collisions between all Trojans $T_{1}$ and $T_{2}$ are considered, it is possible to compute the change in the number of bodies associated to the size bin $k$ of the Trojan population, which is represented by $\Delta N_{k}$.

Once the collisional and dynamical treatment has been developed, the removal rate of small particles due to the action of radiation forces must be included in our analysis. Burns et al. (1979) discussed the main effects of the forces due to solar radiation. They showed that only $\sim 0.1 \mu \mathrm{m}$ particles composed of iron, magnetite, and graphite can be easily ejected from the Solar System by the radiation pressure. Particles smaller and larger than that size are more stable against radiation pressure ejection. However, Burns et al. (1979) indicated that particles of larger sizes drag into the Sun by the Poynting-Robertson effect, while those of smaller sizes are removed by the action of the solar wind. On the other hand, Burns et al. (1979) found that the differential Doppler effect is always less than the Poynting-Robertson effect. According to the works of Burns et al. (1979) and Klačka (1992), the Poynting-Robertson effect is the strongest radiation mechanism that affects the interplanetary dust particles.

Another radiation mechanism able to remove dust particles is the Yarkovsky effect. To analyze the effectiveness of this mechanism in the $L_{4}$ jovian swarm, it is necessary to know the thermal parameters of the jovian Trojans. However, since the understanding of these parameters is still primitive, the Yarkovsky effect is not taken into account in our code. In Sect. 4, we discuss the importance of including the Yarkovsky effect in this task.

The timescale of dust particle removal due to action of the Poynting-Robertson effect is given by

$t_{\mathrm{PR}}=710\left(\frac{r}{\mu \mathrm{m}}\right)\left(\frac{\rho}{\mathrm{g} / \mathrm{cm}^{3}}\right)\left(\frac{R}{\mathrm{AU}}\right)^{2}\left(\frac{1}{1+A}\right) \mathrm{yr}$

(Burns et al. 1979), where $r$ is the radius, $\rho$ the density, $A$ the albedo, and $R$ the distance to the Sun of the dust particle. From this, in a given interval $\Delta t$, the total change in the number of bodies $N_{k}$ associated to a given size bin $k$ of the Trojan population due to collisions and non-collisional removal, is given by

$\left(\Delta N_{k}\right)_{\text {Total }}=\left(\Delta N_{k}-\frac{N_{k}}{t_{\mathrm{PR}}}\right) \Delta t$.

To study the evolution in time of the $L_{4}$ jovian Trojan population, $\Delta t$ is calculated in such a way that the change in the number of objects in any size bin is always less than a given amount, which is generally chosen as $1 \%$ of the original number of bodies.

\section{Results}

The total surface area of the dust cloud existing in the $L_{4}$ jovian swarm can be expressed by

$A_{\text {Dust }}=\frac{\pi}{4} \sum_{D=5 \mu \mathrm{m}}^{500 \mu \mathrm{m}} D^{2} N(D)$ 
where $N(D)$ is the number of particles with diameter $D$. Then, the fractional luminosity of the dust relative to the Sun is given by

$\frac{L_{\text {Dust }}}{L_{\odot}}=\left(\frac{A_{\text {Dust }}}{4 \pi R_{\odot}^{2}}\right)\left(\frac{T}{T_{\odot}}\right)^{4}$,

where $R_{\odot}$ is the radius of the Sun, and $T$ and $T_{\odot}$ are the effective temperatures of the dust particles and the Sun, respectively. To obtain $T$, the incoming solar flux is equaled to the radiated flux from the dust particle, which gives

$$
\begin{aligned}
\pi R^{2}(1-A) S & =4 \pi R^{2} \epsilon \sigma T^{4} \\
T & =\left[\frac{(1-A) S}{4 \epsilon \sigma}\right]^{1 / 4},
\end{aligned}
$$

where $\sigma=5.67 \times 10^{-8} \mathrm{~W} \mathrm{~m}^{-2} \mathrm{~K}^{-4}$ is the Stefan-Boltzmann constant, $\epsilon$ the emissivity (assumed to be 1), $A$ the albedo (assumed to be 0.04 ), and $S$ the solar flux in the position of the particle. Then, $S$ depends on the semimajor axis and can be expressed by the formula

$S=S_{0}\left(\frac{a_{0}}{a}\right)^{2}$,

where $S_{0}=1370 \mathrm{~W} \mathrm{~m}^{-2}$ is the solar constant and $a_{0}$ and $a$ are the semimajor axes of the Earth and the particle under consideration, respectively. For the Jupiter Trojans, $a=5.2 \mathrm{AU}$, and then the effective temperature $T$ of the dust particles in the $L_{4}$ jovian swarm is $121 \mathrm{~K}$. From this and considering that $R_{\odot}=7 \times 10^{5} \mathrm{~km}$ and $T_{\odot}=5800 \mathrm{~K}$, Eq. (12) is written by

$\frac{L_{\text {Dust }}}{L_{\odot}}=3.1 \times 10^{-20}\left(\frac{A_{\text {Dust }}}{\mathrm{km}^{2}}\right)$.

Here, $A_{\text {Dust }}$ depends on the initial population and the collisional parameters of our model. From this, we decided to carry out the numerical simulations 1 and 2, which use the initial populations 1 and 2 defined in Sect. 2.1, respectively, together with the collisional parameters discussed in Sect. 2.2.

Figure 4 shows the estimates of the $L_{4}$ jovian Trojan cumulative size distribution obtained from our two simulations. Our results show waves that propagate from diameters of $\sim 0.1 \mathrm{~km}$ to $\sim 60 \mathrm{~km}$. It is worth noting that the higher the mass of the initial population, the larger the wave amplitude. Thus, the lower the mass of the initial population, the better the fit to the observed size distribution of the $L_{4}$ jovian Trojans. Particularly, the simulation 1 shows a good fit to the observational data of Jewitt et al. (2000).

Figure 5 shows the $L_{4}$ jovian Trojan dust fractional luminosity $L_{\text {Dust }} / L_{\odot}$ as a function of time, obtained from each of our two numerical simulations. From this, we find that the current luminosity of the dust $L_{\text {Dust }}$ in the $L_{4}$ jovian swarm is equivalent in the two different runs. Thus, the time evolution of $L_{\text {Dust }}$ has the characteristics of a diffusion process, since its current value is fairly insensitive to variations in the initial size distribution. On the other hand, our results indicate that the current luminosity of the dust $L_{\text {Dust }}$ in the $L_{4}$ jovian swarm ranges from $\sim 3.2 \times 10^{-8}$ to $3.4 \times 10^{-8} L_{\odot}$.

Then, we tested the role of the Poynting-Robertson effect. Our simulations suggest that the mean collisional lifetimes of the dust particles are shorter than the Poynting-Robertson removal timescale; thus, the evolution of the $L_{4}$ jovian Trojan dust is dominated by collisions, so that, our results do not change significantly when the Poynting-Robertson effect is turned off.

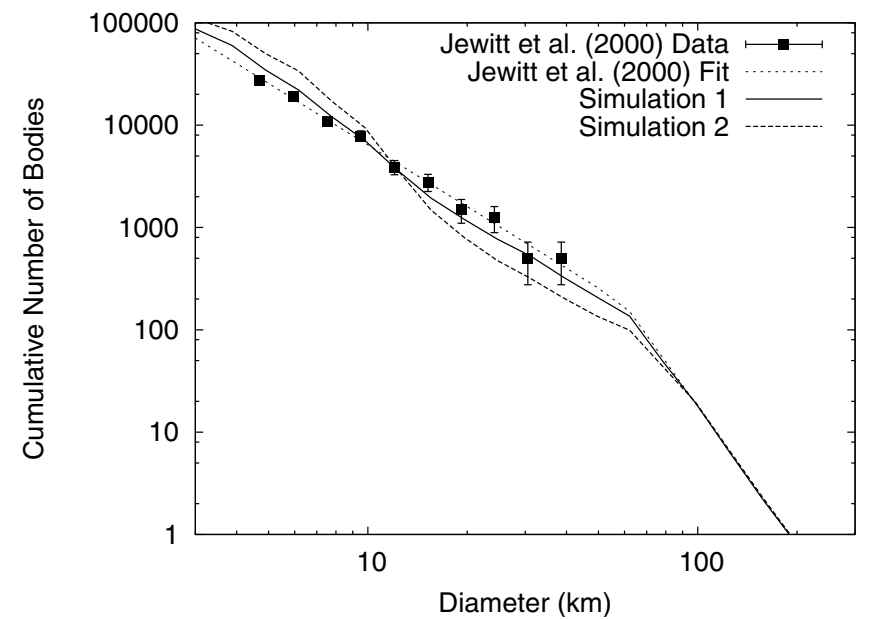

Fig. 4. The estimates of the $L_{4}$ jovian Trojan cumulative size distribution obtained from our two simulations. Observational data derived by Jewitt et al. (2000) and the fit proposed by these authors are given for comparison.

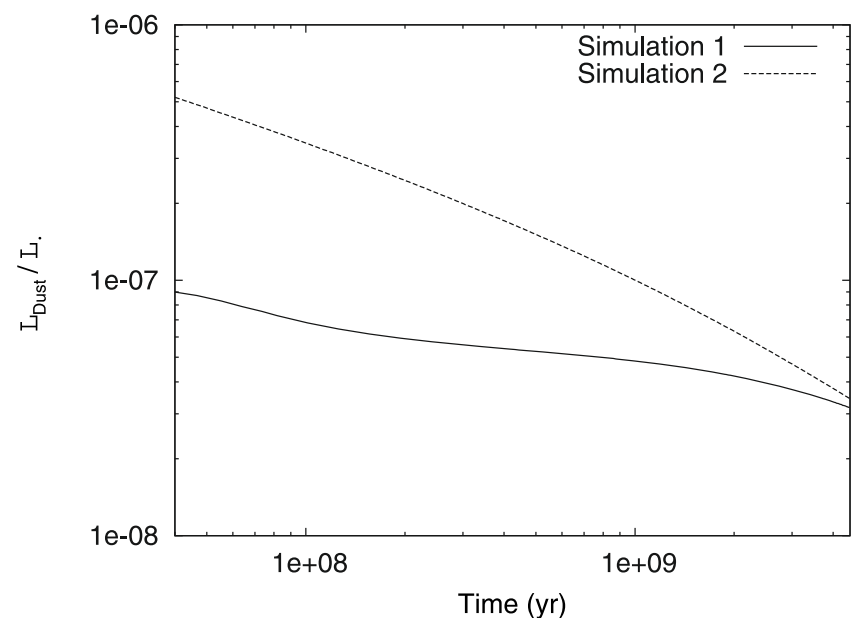

Fig. 5. The $L_{4}$ jovian Trojan dust fractional luminosity $L_{\text {Dust }} / L_{\odot}$ as a function of time, obtained from each of our two numerical simulations.

Finally, we briefly discussed the $L_{5}$ jovian Trojan dust. The intrinsic collision probabilities and the mean impact velocities in the $L_{4}$ and $L_{5}$ swarms have similar values (Dell'Oro et al. 1998), so that the collisional evolution of the populations in both swarms should not be very different. Since the number ratio of $L_{4}$ to $L_{5}$ is 1.3-2.5 (for $D>2 \mathrm{~km}$ ) (Yoshida \& Nakamura 2008), we think that the $L_{4}$ dust production should be somewhat greater than the $L_{5}$ one.

\section{Conclusions}

After applying the numerical code developed by de Elía \& Brunini (2007) to the evolution of the jovian Trojans of the $L_{4}$ swarm, including the effect of the Poynting-Robertson radiation, we analyzed the time evolution of the dust particles with diameters $D \sim 5-500 \mu \mathrm{m}$ in the $L_{4}$ jovian swarm over the age of the Solar System. Our analysis of the size distribution of the $L_{4}$ jovian Trojans sets a constraint on the mass of the initial population. In fact, studying the waves generated in the size distribution, our results show that the lower the mass of the initial population, the better the fit to the observational data derived by Jewitt et al. (2000). 
The studies developed by Dermott et al. (2002) allow us to infer that the luminosity of the inner Solar System dust is estimated at $\sim 10^{-8}-10^{-7} L_{\odot}$. On the other hand, from the size distribution of Kuiper Belt objects analyzed by Stern (1996), Moro-Martín et al. (2008) indicate that the luminosity of the outer Solar System dust is estimated at $\sim 10^{-7}-10^{-6} L_{\odot}$. According to our results, the current luminosity of the dust in the $L_{4}$ jovian swarm is $\sim 3.2 \times 10^{-8}-3.4 \times 10^{-8} L_{\odot}$, which is comparable to the luminosity of the inner Solar System dust and at least one order of magnitude lower than the luminosity of the outer Solar System dust.

Finally, it is worth noting that, since the Yarkovsky effect is not taken account in this work, the current luminosity of the dust in the $L_{4}$ jovian swarm should be somewhat lower than our previous estimates.

Acknowledgements. We acknowledge the valuable discussions with Dr. Romina P. Di Sisto during this work.

\section{References}

Benz, W., \& Asphaug, E. 1999, Icarus, 142, 5

Binzel, R. P., \& Sauter, L. M. 1992, Icarus, 95, 222

Burns, J. A., Lamy, P. L., \& Soter, S. 1979, Icarus, 40, 1

Campo Bagatin, A. 1998, Ph.D. Thesis, University of Valencia, Spain

Campo Bagatin, A., Cellino, A., Davis, D. R., Farinella, P., \& Paolicchi, P. 1994, Planet. Space Sci., 42, 1079

Davis, D. R., \& Weidenschilling, S. J. 1981, Lunar and Planetary Sci. XII, 199

Davis, D. R., Chapman, C. R., Weidenschilling, S. J., \& Greenberg, R. 1985, Icarus, 62, 30

Davis, D. R., Weidenschilling, S. J., Farinella, P., Paolicchi, P., \& Binzel, R. P. 1989, Asteroids II, ed. R. P. Binzel, T. Gehrels, \& M. S. Matthews (Tucson: University of Arizona Press), 805

de Elía, G. C., \& Brunini, A. 2007, A\&A, 475, 375
Dell'Oro, A., Paolicchi, P., Marzari, F., Dotto, E., \& Vanzani, V. 1998, A\&A, 339,272

Dermott, S. F., Durda, D. D., Grogan, K., \& Kehoe, T. J. J. 2002, Asteroids III, ed. W. F. Bottke, A. Cellino, P. Paolicchi, \& R. P. Binzel (Tucson: University of Arizona Press), 423

Farinella, P., Paolicchi, P., \& Zappalà, V. 1982, Icarus, 52, 409

Fernández, Y. R., Sheppard, S. S., \& Jewitt, D. J. 2003, AJ, 126, 1563

Fornasier, S., Dotto, E., Hainaut, O., et al. 2007, Icarus, 190, 622

Holsapple, K. A. 1994, Planet. Space Sci., 42, 1067

Housen, K. R. 1991, Icarus, 94, 180

Housen, K. R., \& Holsapple, K. A. 1990, Icarus, 84, 226

Jewitt, D. C., Trujillo, C. A., \& Luu, J. X. 2000, AJ, 120, 1140

Klačka, J. 1992, Earth, Moon and Planets, 59, 41

Knuth, D. E. 1981, The Art of Computer Programming, Seminumerical Algorithms, 2nd edn. (Reading, Mass.: Addison-Wesley), 2, 688

Landgraf, M., Liou, J.-C., Zook, H. A., \& Grün, E. 2002, AJ, 123, 2857

Love, S. G., \& Brownlee, D. E. 1993, Science, 262, 550

Marzari, F., Farinella, P., Davis, D. R., Scholl, H., \& Campo Bagatin, A. 1997, Icarus, 125, 39

Marzari, F., Scholl, H., Murray, C., \& Lagerkvist, C. 2002, Asteroids III, ed. W. F. Bottke, A. Cellino, P. Paolicchi, \& R. P. Binzel (Tucson: University of Arizona Press), 725

Marzari, F., Tricarico, P., \& Scholl, H. 2003, Icarus, 162, 453

Milani, A. 1993, Celestial Mechanics and Dynamical Astronomy, 57, 59

Milani, A. 1994, Asteroids, Comets, Meteors 1993: Proceedings of the 160th International Astronomical Union, Belgirate, Italy, ed. A. Milani, M. Di Martino, \& A. Cellino, International Astronomical Union (Dordrecht: Kluwer Academic Publishers), Symp., 160, 159

Moro-Martín, A., Wyatt, M. C., Malhotra, R., \& Trilling, D. E. 2008, The Solar System Beyond Neptune, ed. M. A. Barucci, H. Boehnhardt, D. P. Cruikshank, \& A. Morbidelli (Tucson: University of Arizona Press), 465

O’Brien, D. P., \& Greenberg, R. 2005, Icarus, 178, 179

Ryan, E. V., \& Melosh, H. J. 1998, Icarus, 133, 1

Stern, S. A. 1996, AJ, 112, 1203

Szabó, Gy. M., Ivezić, Ž., Jurić, M., \& Lupton, R. 2007, MNRAS, 377, 1393

Tedesco, E. F. 1989, Asteroids III, ed. R. P. Binzel, T. Gehrels, \& M. S. Matthews (Tucson: University of Arizona Press), 1090

Yoshida, F., \& Nakamura, T. 2005, AJ, 130, 2900

Yoshida, F., \& Nakamura, T. 2008, PASJ, 60, 297 